\title{
Meso-scale anisotropic hydrogen segregation near grain-boundaries in polycrystalline nickel characterized by EBSD/SIMS
}

\author{
A. Oudriss ${ }^{\mathrm{a}, *}$, S. Le Guernic ${ }^{\mathrm{a}, \mathrm{b}}$, Z. Wang ${ }^{\mathrm{b}}$, B. Osman Hoch ${ }^{\mathrm{a}}$, J. Bouhattate ${ }^{\mathrm{a}}$, E. Conforto ${ }^{\mathrm{a}}$ \\ Z. Zhu' ${ }^{\text {b D.S. Li }}{ }^{\text {b }}$, X. Feaugas ${ }^{\text {a* }}$
}

\begin{abstract}
${ }^{a}$ LaSIE, CNRS UMR 7356, Université de La Rochelle, av. Michel Crépeau, F-17042 La Rochelle CEDEX 1, France.
\end{abstract}

${ }^{\mathrm{b}}$ Environmental Molecular Sciences Laboratory, Pacific Northwest National Laboratory, Richland, WA 99352, USA.

*Corresponding authors. abdelali.oudriss@univ-lr.fr,xfeaugas@univ-lr.fr

To study anisotropic hydrogen segregation and diffusion in nickel polycrystalline, Secondary Ion Mass Spectrometry (SIMS) and Electron Back Scattered Diffraction (EBSD) are integrated to investigate hydrogen distribution around grain boundaries. Hydrogen distribution in pre-charged samples were correlated with grain boundary character by integrating high-resolution grain microstructure from EBSD inverse pole figure map and low-resolution hydrogen concentration profile map from SIMS. This multimodal imaging instrumentation shows that grain boundaries in nickel can be categorized into two families based on behavior of hydrogen distribution crossing grain boundary: the first one includes random grain boundaries with fast hydrogen diffusivity, showing a sharp gap for hydrogen concentration profile cross the grain boundaries The second family are special $\Sigma 3^{\mathrm{n}}$ grain boundaries with low hydrogen diffusivity, showing a smooth gradient of hydrogen concentration cross the grain boundary. Heterogeneous hydrogen distributions due to grain boundary family revealed by SIMS/EBSD on mesoscale further validate the recent hydrogen permeation data and anisotropic $a b$ initio calculations in nanoscale. The results highlight the fact that grain boundaries character impacts hydrogen distribution significantly.

Keywords: nickel, grain boundaries, hydrogen segregation and diffusion, SEM-EBSD, ToF-SIMS

\section{Introduction}

Understanding hydrogen trapping and diffusivity in materials is very important for a large number of technological applications: storage and distribution of energy, hydrogen permeability, hydrogen embrittlement, etc. [1-3]. Additionally, the durability of materials has become a major concern for the environment and the energy. Hydrogen may easily penetrate and segregate in any material according to its small size, which leads to an early breakdown of the structure. It has been well established that density and distribution of defects have a direct impact on the different states of hydrogen in metal. Consequently, in order to get a better understanding of the behavior of hydrogen inside the materials, the knowledge of distribution of hydrogen is necessary to obtain a complete view of the whole phenomenon at different scales. In a well-annealed polycrystalline metal, two relevant microstructure features can affect the distribution of hydrogen: the grain orientation and the grains boundaries character. Both can be affect the effective diffusivity and solubility. Previous studies in niobium [4] and nickel [5] single crystal demonstrated diffusion anisotropy due to crystallographic orientation. However, anisotropy of hydrogen diffusivity in polycrystalline structure stays unclear. In 
nickel single crystal, the diffusivity depends on crystal orientation [5-9]. In polycrystalline nickel, texture seems to plays an important role in hydrogen permeation [5]. However, role of grain boundary in polycrystalline nickel is not fully investigated yet. Certain orientations are contributing more to hydrogen diffusivity than others, which suggests combinative effects of the distribution of grains orientations and grains boundaries. Recent kinetic Monte Carlo simulations based on data from DFT calculations, demonstrate that diffusion process is determined by grain boundary orientation. In some GBs the diffusivity of hydrogen is lower in normal direction of GB than along GB [10-12]. This leads to the conclusion that hydrogen diffuses faster along GB in the configurations explored in previous simulations. On the contrary, it is generally considered that high concentration of hydrogen can be segregated to the grain boundaries, lowering the strength of atomic bonds and promoted crack propagation when the concentration reaches a critical value. This observation suggests that GBs are preferential sites of high density of defects, which promote hydrogen-trapping processes. This conflicts to the previous simulation revealing the possibility that GBs act as short circuits of diffusion. Extensive works have been performed in the past to identify the role of grain boundaries on the processes of diffusion and trapping around grains boundaries. Contrary to some initial controversies, recent works support the fact that the grains boundaries can play the role of short circuit of diffusion or specific trapping location, depending on the family of the grain boundary [13, 14]. Special grains boundaries namely $\Sigma 3^{\mathrm{n}}$ act as preferential sites of hydrogen trapping; while random grain boundaries act as easy paths for hydrogen diffusion (short circuit of diffusion). These hypotheses are mainly supported by a combination of a large variability of polycrystalline structures and a self-consistent description of the microstructure.

It is important to point out that previous simplified description of polycrystalline microstructure as a composite of isotropic grains-boundaries and grains does not take into account the variable impact of GBs on the anisotropy of diffusion and on the gradient of hydrogen segregation in this complex microstructure. Some works at the atomic scale shows that in the direction of diffusion, the transport path of $\mathrm{H}$ can be assigned to the atomic distribution near GBs [10, 15]. In some cases, it was reported that the diffusion parallel to the grain-boundary is faster than that perpendicular to the grain boundary and the transgranular one. Concerning hydrogen segregation, some prior studies suggest the presence of a gradient of hydrogen concentration near GBs [16-18]. Using microprint technics $[19,20]$ and high-resolution tritium autoradiography [21], a segregation of hydrogen near some GBs was observed, but not for all the GBs [21]. These experimental results imply a variable diffusion behavior in grain boundary, depending on grain boundary character. Birnbaum and coworker suspected this specificity long time ago and tried to prove it using SIMS analyses on some GBs of polycrystals and bi-crystals. These analyses clearly demonstrate for some selected GBs a gradient of hydrogen content with a length path higher than the GBs thickness. But the lack of statistical analyzes and lack of grain boundary categorization has made it difficult to determine the hydrogen distribution and trapping contribution around GBs.

Consequently, the aim of this work is mainly to develop an experimental approach combining EBSD and SIMS to analyze statistically the impact of the GBs character on the hydrogen distribution around the GBs.

\section{Material and methods}

The material used was polycrystalline nickel (purity 99.99\%) with two micrometric grain sizes: $18 \mu \mathrm{m}$ and $45 \mu \mathrm{m}$. Grain size was controlled by thermo-mechanical processing at constant annealing temperature for a predefined time in a controlled atmosphere on cold-drawn round nickel rods provided by Goodfellow [13, 22, 23]. All the samples were prepared by $\mathrm{SiC}$ surface grinding and then electropolished in $\mathrm{H}_{2} \mathrm{SO}_{4} / \mathrm{CH}_{3} \mathrm{OH}$ acid solution [24-26]. Samples were charged in hydrogen and analyses after a specific backing time at room temperature. Hydrogen contents were introduced at room temperature $(298 \mathrm{~K})$ by electrochemical charging for $48 \mathrm{~h}$ at a cathodic current density of -20 $\mathrm{mA} / \mathrm{cm}^{2}$ in a $0.1 \mathrm{M} \mathrm{NaOH}(\mathrm{pH}=13)$ aqueous solution deaerated with an argon pressure of $1.4 \mathrm{bar}$ $[9,13]$. The initial hydrogen concentration is respectively equal to $540 \mathrm{ppm}$ at. and $360 \mathrm{ppm}$ at. for grain sizes of $18 \mu \mathrm{m}$ and $45 \mu \mathrm{m}$, respectively. These concentrations are higher than hydrogen solubility $(6.8 \mathrm{ppm}$ at for $0.1 \mathrm{MPa}$ and $300 \mathrm{~K})$ reported for nickel single crystal at room temperature 
[27]. The oversaturation of hydrogen implies that some GBs promote the hydrogen trapping processes. After hydrogen charging step, several indentation hardness footprints were performed to mark the interest zone for EBSD and SIMS analyses (Figure 1). EBSD in interest zones was carried out using an EDAX/TSL OIM system coupled to a FEI Quanta 200 ESEMFEG scanning electron microscope. An accelerating voltage of $20 \mathrm{kV}$ was chosen to obtain optimum diffraction condition. Automatic beam scanning and the TSL OIM Data Collection 5 software were used to map the crystal orientations on planar sections. The grain boundary character distribution (GBCD) was investigated using crystallographic orientation mapping data as previously proposed [13]. Grain boundaries are categorized into two families: the special GBs $(\Sigma<29)$ corresponding mainly to the $\Sigma 3^{\mathrm{n}}$ in nickel [13] $(\Sigma 3, \Sigma 9$ and $\Sigma 27)$, and random GBs $(\Sigma>29)$. Hydrogen concentration profile maps were calculated from time-of-flight SIMS measurements (TOF.SIMS5, ION-TOF GmbH, Germany). Samples were pumped down in the introduction chamber 12 hours before they were introduced into the analysis chamber. The pressure in the analysis chamber was about $3.4 \times 10^{-9}$ mbar. For each zones studied, the sputtering primary ion beam was a $1.0 \mathrm{keV} \mathrm{Cs}{ }^{+}$beam, which was rastered over a $300 \times 300 \mu \mathrm{m}^{2}$ area during sputtering. A pulsed $25 \mathrm{keV} \mathrm{Bi}^{+}$beam was used for analysis, which was rastered over a $100 \times 100 \mu \mathrm{m}^{2}$ area at the center of the $\mathrm{Cs}^{+}$sputter craters during data acquisition. The pulse frequency of the $\mathrm{Bi}^{+}$beam was $33 \mathrm{kHz}$ and the corresponding current was about $3.8 \mathrm{pA}$. The $\mathrm{H}^{-}$ions were detected because $\mathrm{H}^{-}$signal is more sensitive than $\mathrm{H}^{+}$signal $[28,29]$. After SIMS measurements, the samples were removed from the vacuum, and the depths of sputter craters were measured using a stylus profilometer (Dektak 150, Veeco Instruments, Inc.). A constant sputtering rate was assumed for each depth profiling measurement. The depth of sputter craters was intentionally controlled to be around $270 \pm 20 \mathrm{~nm}$. The first $80 \mathrm{~nm}$ is not taken into account to the summation of hydrogen content used to present a mapping of solute distribution. The utilization of time-of-flight SIMS measurements with a spatial resolution of $400 \mathrm{~nm}$ is not sufficient to improve hydrogen in the grain-boundaries but offers the opportunity to question hydrogen distribution around these ones. Because of the multiple steps required before the SIMS analysis that can lead to large errors, it is impossible to know the exact content of hydrogen for each situation. Thus, only relative values will be presented in this work.

\section{Results and discussion}

The locations when SIMS were performed are allocated in the images from SEM (Fig. 1a) and EBSD (Fig. 1b). A typical mapping of solute content (SIMS data, small color pictures) and corresponding grains orientations and grain boundaries orientations (EBSD data) is shown in figure 1. For the six scans allocated in figure 1, Grain structure is revealed by ${ }^{58} \mathrm{Ni}^{-}$counts (Figure 2a) and ${ }^{1} \mathrm{H}^{-}$ (figure $2 b$ ). Consequently these maps can be easily combined with EBSD images (grain orientation: Figure $2 \mathrm{c}$ and grain boundaries orientation Figure $2 \mathrm{~d}$ ). As it was previously suggested that the number of counts per second is affected by the grain orientation of the crystal face studied. To minimize these effects, the data was normalized using ${ }^{58} \mathrm{Ni}^{-}$. The hydrogen distribution seems depending on grain orientations, which illustrates that boundary conditions of one grain (grain-boundaries) affect the redistribution of hydrogen. To improve this suggestion, we represent the normalized $\mathrm{H}^{-}$signal intensity along a line that intersects different grains orientations and grains boundaries (Figure 3). The specific locations of line scans are identified in EBSD maps. Additionally, using EBSD data, grain boundaries have been identified as special grains boundaries $\left(\Sigma 3^{\mathrm{n}}\right)$ or random grains boundaries (R). Consequently, the comparison between of hydrogen count profile and EBSD map helps to identify hydrogen interaction with GBs. Hydrogen concentration is affected in crossing grain-boundaries as shown in Figure 3. Different situations near GBs are observed, which imposes a statistical approach to characterize the profile of hydrogen around grain-boundaries. The profile of hydrogen around GBs has been investigated qualitatively in relation with the type of GBs for 50 grains boundaries for both grains size. As illustrated on Figures 3 and 4, four kinds of profiles can be reported: gap where instantaneous variation of hydrogen content is observed when we cross the GB, constant profile where no significant modification of hydrogen content is related to both grain around GB, break where the profile evolved when we cross a GB and finally a gradient of hydrogen content for both sides of GB. No significant effect of grain size is observed (Figure 4). Around a large fraction of random grainboundaries, we observed a gap of hydrogen concentration between both adjacent grains (Figures 3a 
and 4a). However, a gradient of hydrogen content for both sides of GB is the main feature observed for the special grain-boundaries (Figures $3 b$ and $4 b$ ). The main experimental results about hydrogen diffusion along random GBs show that this kind of GBs is a short circuit of diffusion in relation with a large excess of free volume [13]. Consequently, it seems that when this process occur along GBs, hydrogen do not cross the GB easily but move along the GB. Thus the random GBs seem do not favor hydrogen homogenization grain to grain. Additionally hydrogen distribution supports the idea that a Random GB accommodates hydrogen more easily than other one and provides diffusion pathways that can determine the preferred direction of hydrogen diffusion along GB. Oudriss et al. have suggested a direct correlation between the excess volume of GBs and an accelerated H diffusion with GB (shortcircuit of diffusion) [13]. More recently, DFT calculations conducted by Di Stephano et al. in nickel, highlight higher hydrogen diffusivity along a 55 GB in relation with an important excess volume. Consequently both results seem support the interpretation of the specific hydrogen distribution (gap) observed around random GBs in present work. To conclude, the GBs with a large excess volume promote a short circuit of diffusion and are not favor to any local hydrogen segregation.

Despite the large variety of special $\Sigma 3^{\mathrm{n}}$, hydrogen gradient around these GBs, suggests that in polycrystalline structure, a strain gradient exist near $\Sigma 3^{\mathrm{n}} \mathrm{GBs}$ with a length scale (which can reach 4 $\mu \mathrm{m})$ significantly higher than GBs thickness $(\approx 2$ to $5 \mathrm{~nm})$. This one should be noted as a surprise, because a large density of defects is necessary to accommodate the misorientation between two adjacent grains which induce long-range internal stresses near GB. To confirm that comment, recently, elastic field was identified for some case around GBs with HRTEM [30]. Additionally, it has been reported previously that hydrogen is deep trapping in $\Sigma 3^{\mathrm{n}} \mathrm{GBs}$ [13], but also a specific location where reverse trapping occurs in relation with the long-range elastic field of dislocation network [13]. Consequently, inhomogeneous hydrogen distribution around $\Sigma 3^{\mathrm{n}} \mathrm{GBs}$ can be the result to an elastic lattice distortion around GB more than the specificity of the local geometric structure. According to recent work [13], this kind of GBs promotes local hydrogen segregation, but do not enhance hydrogen diffusivity.

Twin is a specific configuration of $\Sigma 3^{\mathrm{n}}$ GBs, where any defects are necessary to accommodate the misorientation for coherent twin GBs. In this case, we do not observe a gradient profile (Figure 3c) and the concentration of hydrogen is lower than the one obtained in grain. Consequently, this closepacked grain boundary configuration acts as a two-dimensional barrier to hydrogen diffusion, consistent with the previous DFT studies $[15,32]$. According to recent DFT calculations perform on $\Sigma 3$ symmetric twin grain boundary in nickel, the segregation energy is negligible for this GB, the diffusion parallel to the GB plane is equal to its in the grain interior but the diffusion across the GB plane seems to be associated with a significantly higher barrier. Consequently, it is not suppressing to observe a low concentration of hydrogen in a twin and no segregation. In opposite, actually any DFT results exist about more defective $\Sigma 3^{\text {n }}$ GBs.

\section{Conclusion}

To conclude, according to our SIMS analyses, hydrogen distribution in "stationary state" in polycrystalline pure nickel depends on grain-boundaries character. Two main situations were observed for hydrogen in relation with the GBs populations. These results seem confirm our previous work, which support a high diffusivity along random GBs and a specific location of trapping kinetic around special GBs [13]. Despite these new relevant results, it is clear concluded that actually it stays difficult to demonstrate that hydrogen distribution result to a heterogeneous behaviour of diffusion and segregations processes or both.

In future works, this approach can be extended to question the impact of the adjacent grain boundaries orientation of GBs on the hydrogen segregation and mobility. As it was recently demonstrate for carbon-steel system [31], the present contribution is a new illustration of the opportunity to use multimodal characterization technique (SIMS and EBSD) to question solutes segregation in relation with metallurgical state

\section{Acknowledgments}


This work was funded by the French Agency (ANR) under contract No ANR-13-JS09-0015-01. The authors would like to thank Mrs. Christelle Rébéré and Mr. Cyril Berziou (LaSIE, UMR CNRS 7356, ULR, F-17000 La Rochelle, France) for their technical support. The SEM-EBSD measurements were performed at Laboratoire des Sciences de L'Ingénieur pour l'Environnement (LaSIE-CNRS) using microscopy center facilities. The ToF-SIMS measurement was performed at Environmental Molecular Sciences Laboratory (EMSL), a national scientific user facility sponsored by the Department of Energy's Office of Biological and Environmental Research located at PNNL.

\section{References}

[1] M. F. Ashby, J. P. Hirth, Perspectives in Hydrogen in Metals. Collected Papers on the Effect of Hydrogen on the Properties of Metals and Alloys, first ed., Pergamon Press, Oxford, New York 1986. [2] R.P. Gangloff, B.P. Somerday, Gaseous Hydrogen Embrittlement of Materials in Energy Technologies, Woodhead Publishing, Cambridge, U.K. 2012.

[3] I.M. Robertson, P. Sofronis, A. Nagao, M.L. Martin, S. Wang, D.W. Gross, K.E. Nygren, Metall. Mater. Trans. A 46 (2015) 2323-2341.

[4] G. Kistner, R. Rubin, I. Sosnowska, Phys. Rev. Letter 27 (1971) 1576-1577.

[5] Y. Cao, H. L. Li, J. A. Szpunar, W.T. Shmayda, Mater. Science Forum, 408-412 (2002) 1139.

[6] A. M. Brass and A. Chanfreau, Acta Mater. 44 (1996) 3823-3831.

[7] A. M. Brass, A. Chanfreau, and J. Chene, in Hydrogen Effects on Materials Behavior, eds N. R. Moody and A. W. Thompson, Minerals, Metals, and Materials Society, Warrendale, PA, 1990.

[8] Y. Ebisuzaki, W. J. Kass, M. O'Keeffe, J. Chem. Phys. 46 (1967) 1378-1381

[9] A. Oudriss, J. Creus, J. Bouhattate, C. Savall, B. Peraudeau, X. Feaugas, Scripta Mater. 66 (2012) 37-40.

[10] A. Pedersen, H. Jonsson, Acta Mater. 57 (2009) 4036-4045.

[11] W. Yongli, X. Liangyin, L. Shi, Acta Metall. Sinica 27 (2014) 615-620.

[12] Y. A. Du, J. Rogal, R. Drautz, Phys. Rev. B 86 (2012) 174110.

[13] A. Oudriss, J. Creus, J. Bouhattate, E. Conforto, C. Berziou, C. Savall, X. Feaugas, Acta Mater. 60 (2012) 6814-6828.

[14] E. Legrand, J. Bouhattate, X. Feaugas, S. Touzain, H. Garmestani, M. Khaleel, D.S. Li, Comp. Mater. Science 71 (2013) 1-9.

[15] Y. A. Du, L. Ismer, J. Rogal, T. Hickel, J. Neugebauer, and R. Drautz, Phys. Rev. B 84 (2011) 144121.

[16] Y. Park, J. Lu, G. Rozgonyi, Electron. Mater. Lett.. 6 (1) (2010) 1-5.

[17] H. Fukushim, H. K. Birnbaum, Acta metal. 32 (1984) 851-859.

[18] B. Ladna, H. K. Birnbaum, Acta metal. 35 (1987) 2535-2542.

[19] T. Ohmisawa S. Uchiyamaa, M. Nagumoa, J. Alloys and Comp. 356-357 (2003) 290-294.

[20] G. Katano, K. Ueyama, M. Mori, J. Mater. Sci. 36 (2001) 2277-2286.

[21] M. Aucouturier, Journal de Physique Colloques, 43 (1982) C6-175-C6-186.

[22] X. Feaugas, H. Haddou. Metall. Trans. A 34 (2003) 2329-2340.

[23] X. Feaugas, H. Haddou, Phil. Mag., 87 (2007) 989-1018.

[24] M. Sahal, J. Creus, R. Sabot, X. Feauga, Scripta Mater. 51 (2004) 869-873.

[25] H. El Alami, J.Creus, X.Feaugas, Electrochim. Acta. 51 (2006) 4716-4727.

[26] C. Lekbir, J. Creus, R. Sabot, X. Feaugas. Mater. Sci. Eng. A 578 (2013) 24-34.

[27] Y. Fukai, The Metal-Hydrogen System: Basic Bulk Properties, Springer-Verlag, Berlin, 2006.

[28] Z. Zhu, V. Shutthanandan. Surf. Interface Anal. 44 (2012) 89-93.

[29] Z. Zhu, V. Shutthanandan, M. Engelhard. Surf. Interface Anal. 44 (2012) 232-237.

[30] C. W. Zhaoa, Y. M. Xing, P. C. Bai, J. F. Hou, X. J. Dai. Physica B 403 (2008) 1838-1842.

[31] K. S. Choi, Z. Zhu, X. Sun, E. De Moor, M. D. Taylor, J. G. Speerb, D.K. Matlock, Scripta Mater. 104 (2015) 79-82.

[32] D. Di Stefano, Ma. Mrovec, C. Elsässer, Acta Materialia 98 (2015) 306-312.

\section{Figure captions}


Figure 1: a) SEM and b) EBSD maps showing the localization of the six SIMS maps in both.

Figure 2: Signal intensity for ${ }^{58} \mathrm{Ni}^{-}$(a) and ${ }^{1} \mathrm{H}^{-}$(b). Mapping of grains orientations (c) and grain boundaries families (d). (Sample with grain size of $45 \mu \mathrm{m}$, scan number 3).

Figure 3: Some examples of possible correlation between EBSD and SIMS analyses. : Line profile position and normalized ${ }^{1} \mathrm{H}^{-}$signal intensity (at right) versus the position along the line (at left). (a) Random, (b) $\Sigma 3^{\mathrm{n}}$ and (c) Hydrogen distribution around a twin.

Figure 4: Fraction of grain-boundaries, which present one kind of hydrogen distribution. (a) Random GBs and (b) $\Sigma 3^{\text {n }}$ GBs. 

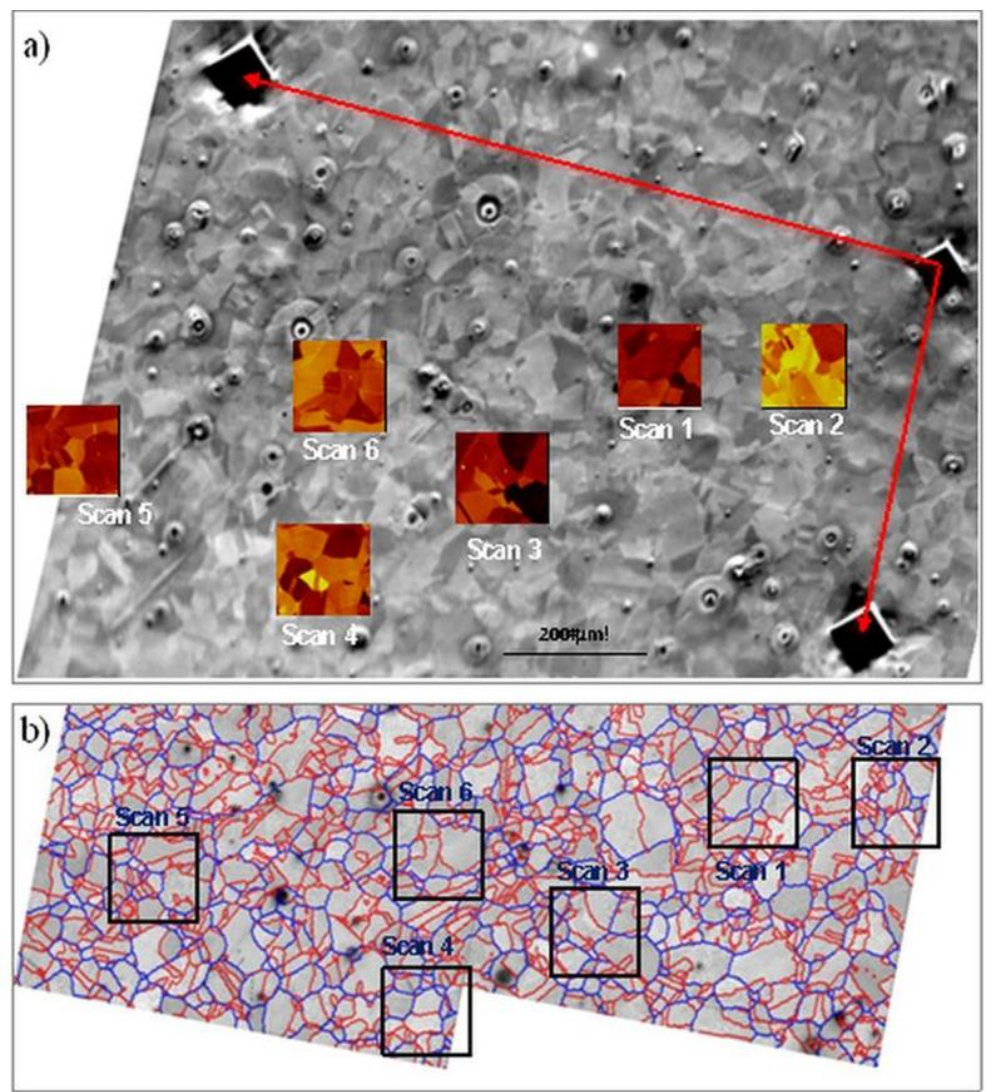

Figure 1: a) SEM and b) EBSD maps showing the localization of the six SIMS maps in both. 

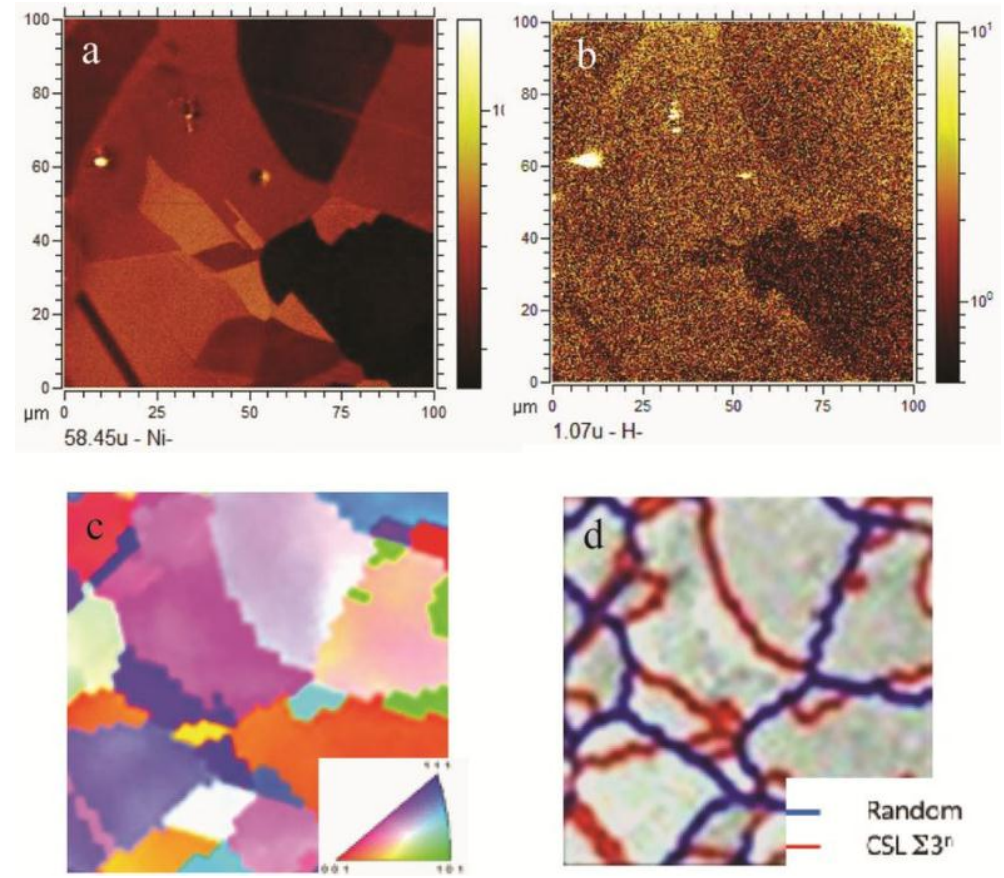

Figure 2: Signal intensity for ${ }^{58} \mathrm{Ni}^{-}$(a) and ${ }^{1} \mathrm{H}^{-}$(b). Mapping of grains orientations (c) and grain boundaries families (d). (Sample with grain size of $45 \mu \mathrm{m}$, scan number 3 ). 

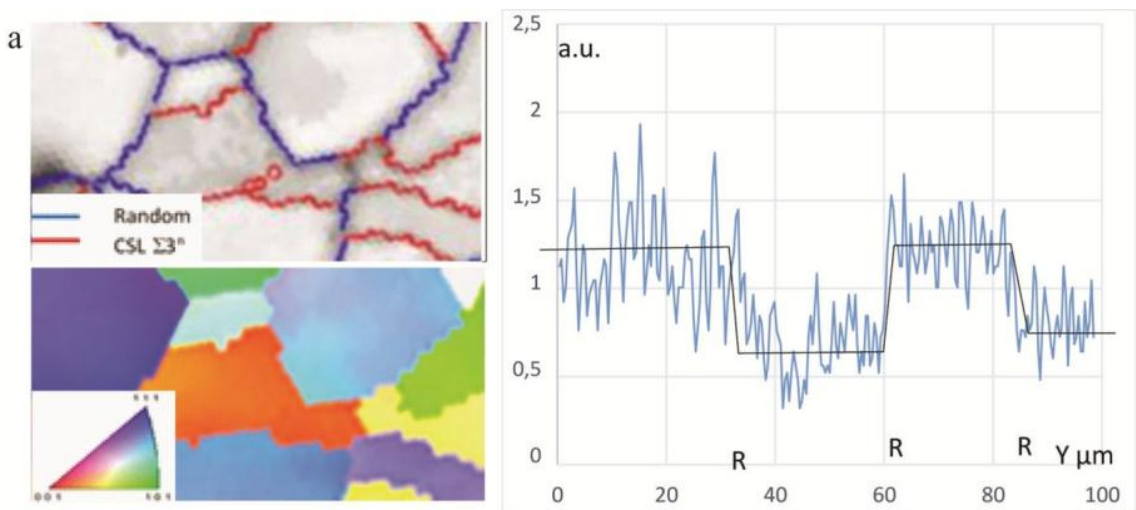

b
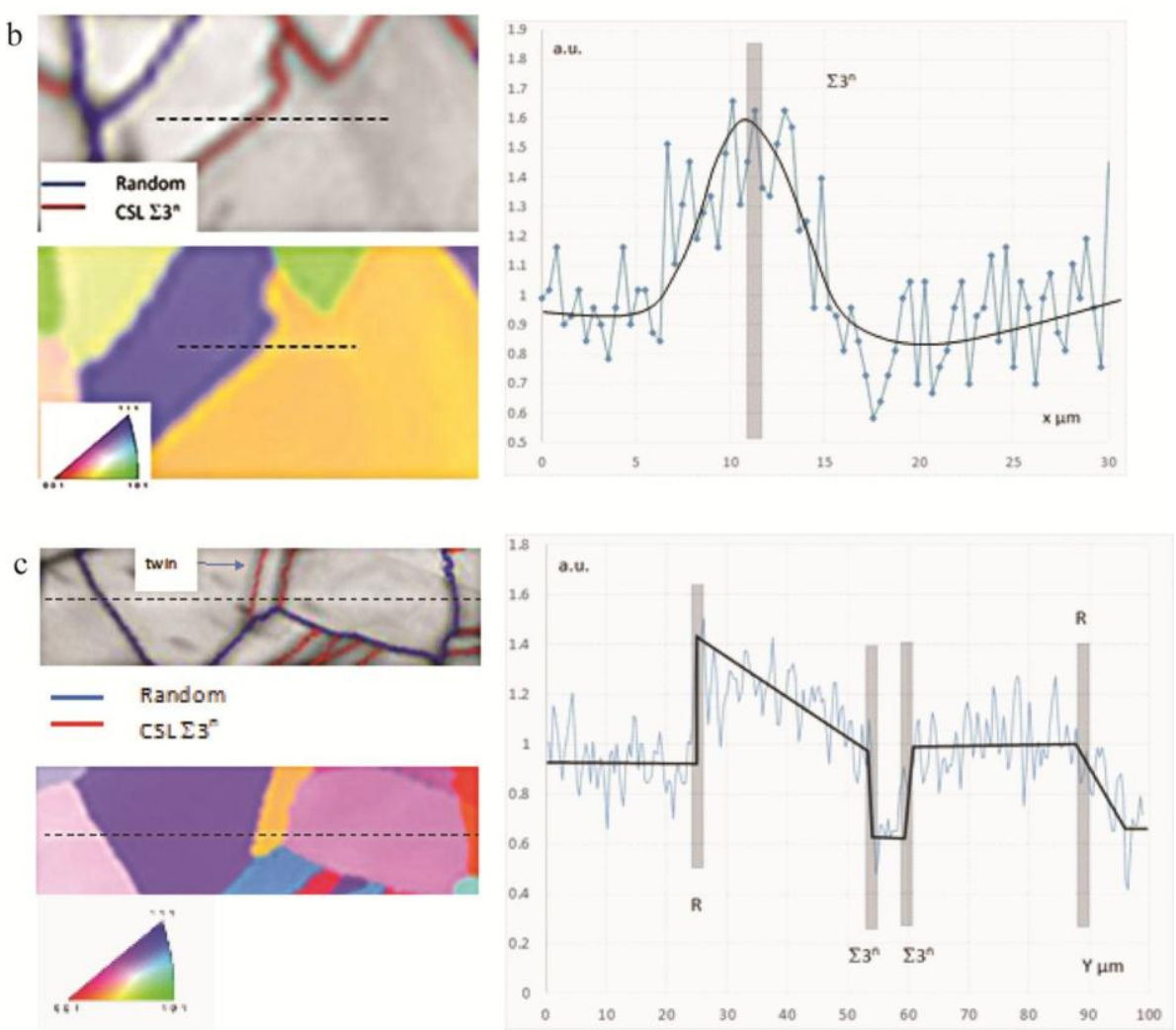

Figure 3: Some examples of possible correlation between EBSD and SIMS analyses. : Line profile position and normalized ${ }^{1} \mathrm{H}^{-}$signal intensity (at right) versus the position along the line (at left). (a) Random, (b) $\Sigma 3^{\mathrm{n}}$ and (c) Hydrogen distribution around a twin. 

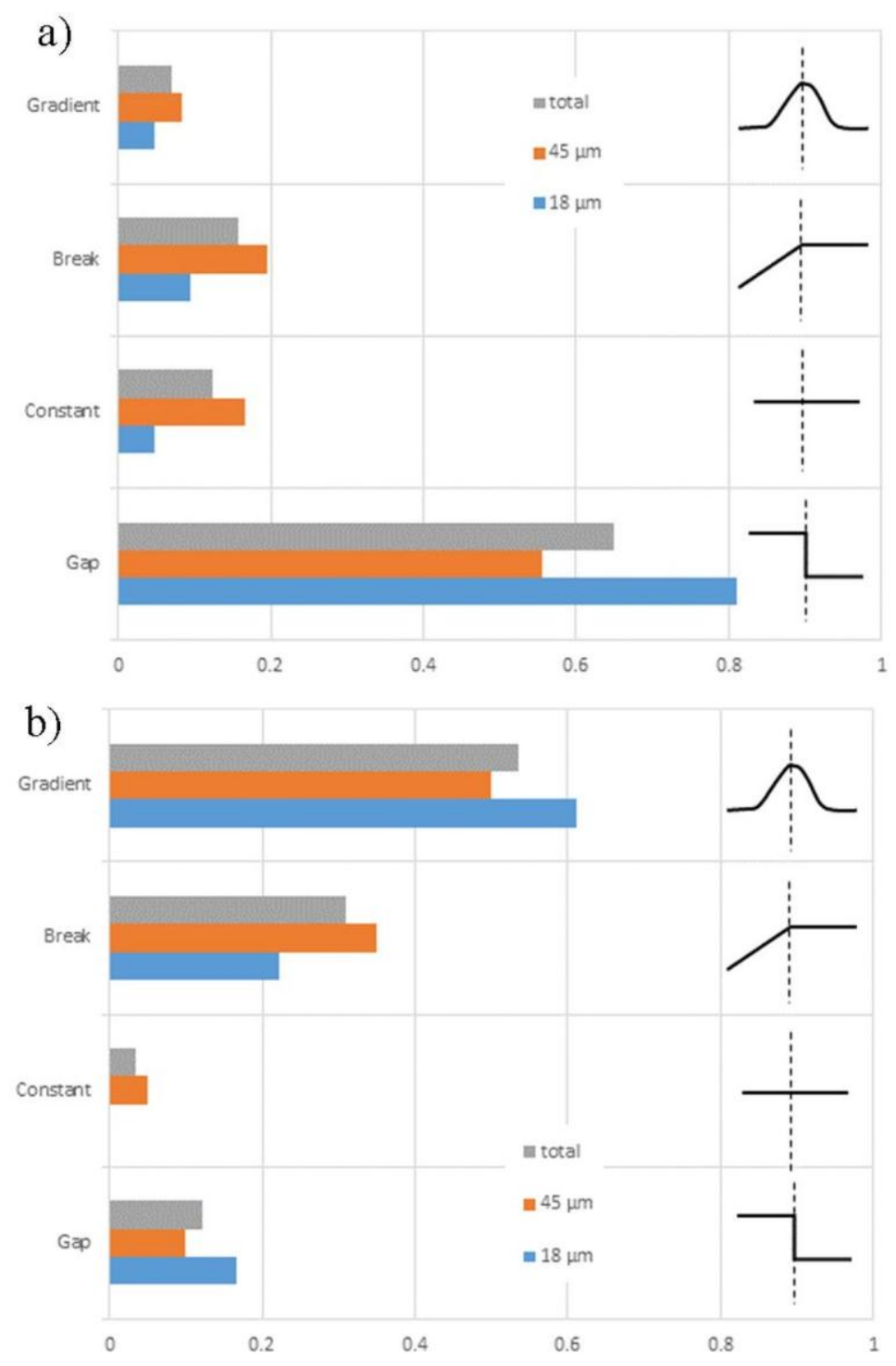

Figure 4: Fraction of grain-boundaries, which present one kind of hydrogen distribution. (a) Random GBs and (b) $\Sigma 3^{\mathrm{n}}$ GBs. 


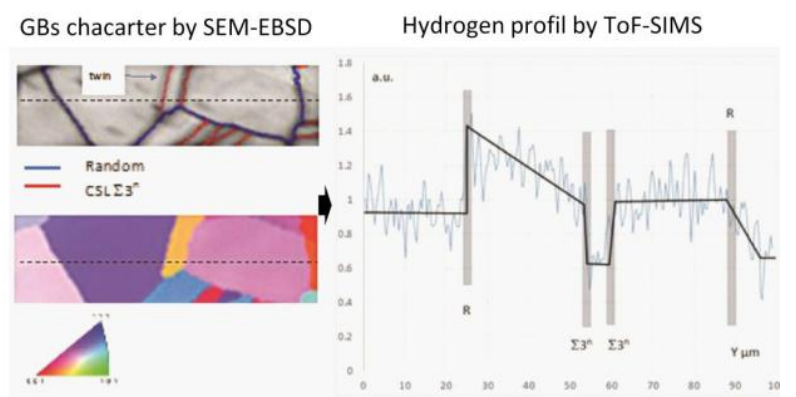

